\title{
Simple Test for Hidden Variables in Spin-1 Systems
}

\author{
Alexander A. Klyachko, ${ }^{*}$ M. Ali Can, ${ }^{\dagger}$ Sinem Binicioğlu, and Alexander S. Shumovsky \\ Faculty of Science, Bilkent University, Bilkent, Ankara, 06800 Turkey
}

(Received 1 June 2007; published 11 July 2008)

\begin{abstract}
We resolve an old problem about the existence of hidden parameters in a three-dimensional quantum system by constructing an appropriate Bell's type inequality. This reveals the nonclassical nature of most spin-1 states. We shortly discuss some physical implications and an underlying cause of this nonclassical behavior, as well as a perspective of its experimental verification.
\end{abstract}

DOI: 10.1103/PhysRevLett.101.020403

PACS numbers: 03.65.Ud, 03.65.Ta

The concept of quantum entanglement, as well as the prospect of its applications in quantum computing, has attracted a great deal of attention [1]. No doubt, its most striking manifestation is quantum nonlocality, understood here as a correlation beyond light cones of spatially separated quantum systems, where no classical interaction between them is possible. However, for quantum computation the magic ability of entanglement to bypass constraints imposed by the so-called classical realism is far more important. The latter is understood here as the existence of hidden parameters, or equivalently a joint probability distribution of all involved quantum observables. This property of entanglement can be modeled by no classical device, which emphasizes a qualitative distinction between classical and quantum information processing, beyond a mere difference in their computational power.

Following Bell's seminal works [2], the nonclassical behavior is usually detected by violation of certain inequalities, collectively named Bell's conditions. Their experimental test [3] left little or no doubt that entangled states indeed override the classical constraints, in spite of everlasting search for possible loopholes [4].

Initially Bell justified his constraints by locality argument that spacelike separated quantum systems can not affect each other. This approach neither excludes the existence of nonlocal hidden parameters nor can be applied to local systems. In this Letter we provide a test for hidden variables in a local spin-1 system, where the original Bell's approach clearly fails. We found that every spin state is nonclassical, except for coherent one $|1\rangle_{\ell}$ with spin projection 1 onto some direction $\ell$.

To elucidate the physical difference between the coherent state $|1\rangle$ and its antithetical counterpart $|0\rangle$, called neutrally polarized spin state, consider a decay of spin-1 system into two spin- $1 / 2$ components. The resulting two particle state must be symmetric under the exchange of the components and preserve the angular momentum. As a result, the coherent state $|1\rangle$ decays into the separable one $|\uparrow \uparrow\rangle$, while the neutrally polarized state $|0\rangle$ yields the maximally entangled Bell state $\frac{1}{\sqrt{2}}(|\uparrow \downarrow\rangle+|\downarrow \uparrow\rangle)$.

The problem we address here is whether one can detect something nonclassical in the state $|0\rangle$ before the decay.
Recall that by the Kochen-Specker theorem [5] every spin1 state is incompatible with the so-called context-free hidden variables model. The latter entertains the notion of "hidden value" of an observable $A$ revealed by its measurement and independent of a measurement of any other observable $B$ commuting with $A$. Bell found no physical ground for this hypothesis and eventually abandoned it in favor of inequalities based on locality rather than noncontextuality. However, on the way he switched from spin-1 system to system of two qubits, leaving the problem of existence of contextual hidden parameters in spin-1 system open. We resolve this problem below.

Our approach to hidden variables in spin systems is similar to that of Fine [6] for two qubits with two measurements $A_{1}, A_{2}$ and $B_{1}, B_{2}$ at sites $A$ and $B$, respectively. The hidden variables provide a joint probability distribution of all four observables $A_{1}, A_{2}, B_{1}, B_{2}$, compatible with the distributions of commuting pairs $A_{i}, B_{j}$ predicted by quantum mechanics and available for experimental verification. The arising general problem of existence of a joint probability distribution of random variables $x_{1}, x_{2}, \ldots, x_{n}$, compatible with given partial distributions of some of them, is known as the marginal problem [7]. Geometrically it amounts to the existence of a "body" in $\mathbb{R}^{n}$ of a non-negative density with given projections onto some coordinate subspaces.

The problem was settled in the early 1960s [8]. Applying the solution to observables $A_{i}, i=1,2, \ldots, n$ in an arbitrary finite quantum system we arrive at the following ansatz for testing classical realism [9]. Let $a_{i}$ be a variable describing all possible outcomes of the observable $A_{i}$. We will use the shortcuts $A_{I}$ for the subset of observables $A_{i}$, $i \in I$, and $a_{I}$ for the corresponding subset of variables $a_{i}$, $i \in I$. Consider now a non-negative function of the form

$$
\sum_{A_{I} \text { commute }} f_{I}\left(a_{I}\right) \geq 0
$$

and assume the existence of a hidden distribution of all variable $a_{1}, a_{2}, \ldots, a_{n}$ compatible with the distributions of commuting observables $a_{I}$ predicted by quantum mechanics. Then, taking the expectation value of Eq. (1) with respect to the hidden distribution, we arrive at the Bell- 
type inequality for testing classical realism

$$
\sum_{A_{I} \text { commute }}\left\langle\psi\left|f_{I}\left(A_{I}\right)\right| \psi\right\rangle \geq 0
$$

It turns out that these inequalities are also sufficient for the existence of hidden variables [8]. To make this criterion effective, observe that the set of all functions given by Eq. (1) form a polyhedral cone, called Vorob'ev-Kellerer cone, and the conditions (2) should be checked for its extremal edges only. The latter can be routinely found using an appropriate software, e.g., CONVEX package [10].

As a result, we end up with a finite set of inequalities that are necessary and sufficient for an extension of the partial distributions of commuting observables $A_{I}$ to a hidden distribution of all observables $A_{i}$, commuting or not. The latter can be modeled by classical means like tossing dice. This makes the quantum system indistinguishable from a classical one.

Let us separate the extremal edges generated by a single function $f_{I}\left(a_{I}\right) \geq 0$ vanishing everywhere except one point. The corresponding Bell inequality is vacuous and we call such extremal functions trivial.

For two qubits the ansatz gives eight nontrivial extremal functions. The respective constraints can be obtained from Clauser-Horne-Shimoni-Holt (CHSH) inequality [11]

$$
\left\langle A_{1} B_{1}\right\rangle+\left\langle A_{1} B_{2}\right\rangle+\left\langle A_{2} B_{1}\right\rangle-\left\langle A_{2} B_{2}\right\rangle \leq 2,
$$

by spin flips $A_{i} \mapsto \pm A_{i}, B_{j} \mapsto \pm B_{j}$. This criterion for existence of hidden parameters was first proved by Fine [6].

Returning to spin-1 system, consider a cyclic quintuplet of unit vectors $\ell_{i} \perp \ell_{i+1}$ with the indices taken modulo 5, see Fig. 1. We call it a pentagram. The orthogonality implies that squares of spin projection operators $S_{\ell_{i}}$ onto directions $\ell_{i}$ commute for successive indices $\left[S_{\ell_{i}}^{2}, S_{\ell_{i+1}}^{2}\right]=$ 0 . We find it more convenient to deal with the observables $A_{i}=2 S_{\ell_{i}}^{2}-1$ taking values $a_{i}= \pm 1$. They satisfy the

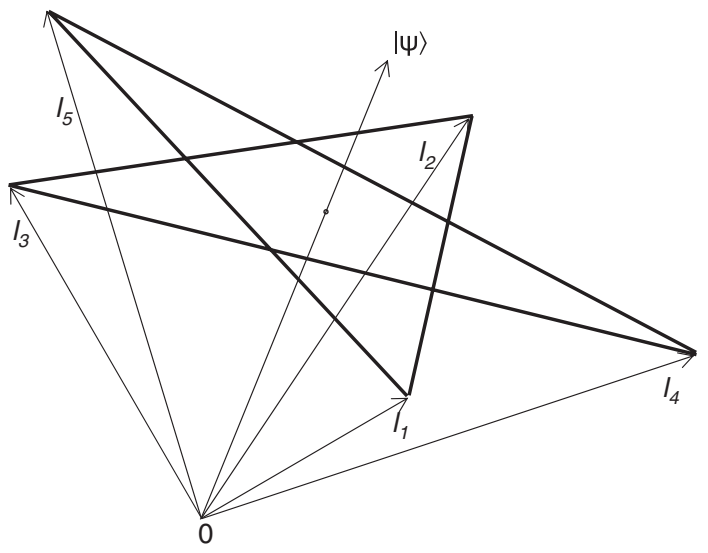

FIG. 1. Regular pentagram defined by cyclic quintuplet of unit vectors $\ell_{i} \perp \ell_{i+1}$. State vector $|\psi\rangle$ is directed along the symmetry axis of the pentagram. following inequality:

$$
a_{1} a_{2}+a_{2} a_{3}+a_{3} a_{4}+a_{4} a_{5}+a_{5} a_{1}+3 \geq 0 .
$$

Indeed, the product of the monomials in the left-hand side is equal to 1 ; hence, at least one term is equal to +1 , and the sum of the rest is no less than -4 .

Assuming now the existence of a hidden distribution of all observables $a_{i}$, and taking the respective expectation value of Eq. (4) we arrive at the inequality

$$
\left\langle A_{1} A_{2}\right\rangle+\left\langle A_{2} A_{3}\right\rangle+\left\langle A_{3} A_{4}\right\rangle+\left\langle A_{4} A_{5}\right\rangle+\left\langle A_{5} A_{1}\right\rangle \geq-3,
$$

that can be recast into the form

$$
\left\langle S_{\ell_{1}}^{2}\right\rangle_{\psi}+\left\langle S_{\ell_{2}}^{2}\right\rangle_{\psi}+\left\langle S_{\ell_{3}}^{2}\right\rangle_{\psi}+\left\langle S_{\ell_{4}}^{2}\right\rangle_{\psi}+\left\langle S_{\ell_{5}}^{2}\right\rangle_{\psi} \geq 3
$$

using the identity $A_{i} A_{i+1}=2 S_{\ell_{i}}^{2}+2 S_{\ell_{i+1}}^{2}-3$ easily derived from Eq. (6) below. We call it the pentagram inequality.

Initially the left-hand side of the inequality (4) was found by a computer as an extremal function of the Vorob'ev-Kellerer cone. The other nontrivial extremal functions can be obtained from it by flips $a_{i} \mapsto \pm a_{i}$. They, however, add no new physical constraints. For example, a single flip $A_{i} \mapsto-A_{i}$ in Eq. (5) yields the inequality $\left\langle S_{\ell_{i}}^{2}\right\rangle \leq\left\langle S_{\ell_{i-2}}^{2}\right\rangle+\left\langle S_{\ell_{i+2}}^{2}\right\rangle$. Since in the pentagram $\ell_{i-2} \perp \ell_{i+2}$, then $S_{\ell_{i-2}}^{2}+S_{\ell_{i+2}}^{2}+S_{n_{i}}^{2}=2$ for some direction $n_{i} \perp \ell_{i \pm 2}$, and the inequality becomes trivial $\left\langle S_{\ell_{i}}^{2}\right\rangle+$ $\left\langle S_{n_{i}}^{2}\right\rangle \leq 2$.

In summary, the pentagram inequality, in contrast to the Kochen-Specker theorem, provides a test for arbitrary hidden variables model, context free or not. Moreover, the inequality is sufficient for the existence of such a model for the observables $S_{\ell_{i}}^{2}$. In addition, it reduces the number of involved spin projection operators from 31 , as in the best-known noncontextual test due to Conway and Kochen, to 5. As a drawback, the pentagram criterion is state dependent.

A more careful analysis shows that there is no hidden variables test for the three-dimensional quantum system with less than 5 observables. Furthermore, every such test with 5 observables $A_{i}$ by an appropriate scaling $A_{i} \mapsto$ $\alpha_{i} A_{i}+\beta_{i}$ can be reduced to the inequality (5) for a complex pentagram $\ell_{i} \perp \ell_{i+1} \in \mathcal{H}$ and $A_{i}=1-2\left|\ell_{i}\right\rangle\left\langle\ell_{i}\right|$, cf. Eq. (6). We will provide the details elsewhere.

For further analysis of the pentagram inequality it is convenient to identify Hilbert space of spin-1 particle with complexification $\mathcal{H}=\mathbb{E}^{3} \otimes \mathbb{C}$ of the physical Euclidean space $\mathbb{E}^{3}$. The spin group $\mathrm{SU}(2)$, locally isomorphic to $\mathrm{SO}(3)$, acts on $\mathcal{H}$ by rotations in $\mathbb{E}^{3}$. The cross product $[x, y]=x \times y$ turns Euclidean space $\mathbb{E}^{3}$ into Lie algebra $\mathfrak{S} \mathfrak{H}(2)$ and allows us to express the spin projection operator as follows: $S_{\ell} \psi=i[\ell, \psi]$. It has three eigenstates, one real $|0\rangle_{\ell}=\ell$ and two complex conjugate $| \pm 1\rangle_{\ell}=$ $(m \pm i n) / \sqrt{2}$, where $\{\ell, m, n\}$ is as an orthonormal basis 
in $\mathbb{E}^{3}$. So in this picture the neutrally polarized spin state $|0\rangle_{\ell}$ is represented by real vector $\ell \in \mathbb{E}^{3}$. The operators $A_{i}$ are now given by the equation

$$
A_{\ell}=I-2|\ell\rangle\langle\ell|=2 S_{\ell}^{2}-I,
$$

that allows us to recast the pentagram inequality into the geometrical form

$$
\sum_{k \bmod 5}\left|\left\langle\ell_{k} \mid \psi\right\rangle\right|^{2} \leq 2
$$

Let us test it for a neutrally polarized spin state represented by a real vector $\psi$ directed along fivefold symmetry axis of a regular pentagram, see Fig. 1. A simple calculation shows that in this case $\left|\left\langle\ell_{k} \mid \psi\right\rangle\right|^{2}=\cos ^{2} \widehat{\ell_{k} \psi}=\frac{1}{\sqrt{5}}$, which violates the pentagram inequality

$$
\sum_{k \bmod 5}\left|\left\langle\ell_{k} \mid \psi\right\rangle\right|^{2}=\sqrt{5} \approx 2.236>2 .
$$

Thus the neutrally polarized spin states are nonclassical. If one believes in invariance of physical laws with respect to rotations around $\psi$ axis, then the distributions of spin projections onto all 5 directions $\ell_{k}$ of the regular pentagram must be the same, and only one of them should be actually measured to refute any hidden variables model. Such a reduction is possible only for the neutrally polarized spin states, that exhibit the most extreme nonclassical behavior. One cannot achieve that high symmetry in the two qubits setting (3), and has to switch the spin projection directions at both sites which may create a loophole [4].

As an example of the spin-1 system of some physical interest, consider a $p$ electron in an atom or a molecule with respect to its orbital momentum, equal to 1 , and disregarding the spin. In the coherent state $|1\rangle$, with orbital momentum 1 in some direction, the electron density looks like a classical Kepler orbit, while in the neutrally polarized state $|0\rangle$ the electron splits itself into two blobs separated by a plane of zero electron density. In the latter case the electron is hopping between these two regions never crossing the plane. This state, known as $p$ orbital, plays a crucial role in chemistry. The nonclassical nature of this state, and the whole chemistry, can be detected by the pentagram inequality.

It may be also instructive to look into the meaning of the pentagram inequality for a composite spin-1 system formed by two components $A, B$ of spin- $1 / 2$. In this setting $S_{\ell}=S_{\ell}^{A}+S_{\ell}^{B}$ and by substitution into Eq. (6) we get a two-component version of the pentagram inequality valid for symmetric states of two qubits

$$
\left\langle A_{1} B_{1}\right\rangle+\left\langle A_{2} B_{2}\right\rangle+\left\langle A_{3} B_{3}\right\rangle+\left\langle A_{4} B_{4}\right\rangle+\left\langle A_{5} B_{5}\right\rangle \geq 1,
$$

where we use the notations $A_{i}=2 S_{\ell_{i}}^{A}, B_{j}=2 S_{\ell_{j}}^{B}$ to facilitate a comparison with CHSH inequality (3). The crucial difference between them is in the directions of the spin projection measurements at sites $A$ and $B$ which for the pentagram version are always the same. This allows us to detect entanglement in closely tight systems, like atoms or molecules, where one may not see the separate components. The latter conclusion holds true even if the components $A, B$ do not exist outside the system, like quarks or quasiparticles.

These observations may suggest that the nonclassical behavior of the spin- 1 system detected by the pentagram inequality originates from entanglement of its internal degrees of freedom, whatever their physical nature could be. This is in line with the Majorana picture of a high spin state as a symmetric state of $2 S$ virtual spin- $1 / 2$ components readily visualized as a configuration of $2 S$ points in Bloch sphere [12]. A proper name for this nonclassical effect would be spin state entanglement [13].

The above discussion may also clarify physical meaning of a more general notion of "entanglement beyond subsystems," promoted by some research groups $[9,14,15]$.

Observe that every state of a general spin- 1 system can be transformed by a unitary rotation into the canonical form

$$
\psi=m \cos \varphi+i n \sin \varphi,
$$

where $m, n$ are two fixed unit orthogonal vectors in $\mathbb{E}^{3}$. Intrinsic properties of $\psi$ are determined by the parameter $0 \leq \varphi \leq \frac{\pi}{4}$. For example, Wootters's concurrence $c(\psi)$ [16] of spin state $\psi$, considered as a symmetric state of two qubits, is equal to $\cos 2 \varphi$ and coincides with a measure of entanglement for spin states introduced in [17]. The extremal values $c=0$ and $c=1$ correspond to the coherent $|1\rangle$ and the neutrally polarized $|0\rangle$ spin states, respectively.

Note that a regular pentagram can detect nonclassical nature of a spin- 1 state $\psi$ only for $c(\psi)>\frac{1}{\sqrt{5}}$. For a state with a smaller positive concurrence one has to use an appropriate skew pentagram. On the other hand, coherent states pass the test for any pentagram, and hence they are the only classical spin-1 states. We refer for details to Ref. [15].

As a convenient physical model of spin- 1 system suitable for experimental study consider a single mode biphoton, i.e., a pair of photons in the same spatiotemporal mode, so that they differ only in polarization [18]. The photons obey Bose statistics; hence, their polarization space is spanned by the symmetric triplet

$$
\left.\left.|\circlearrowleft\rangle\rangle, \quad \frac{1}{\sqrt{2}}(|\cup \circlearrowright\rangle+|\circlearrowleft\rangle\rangle\right), \quad|\circlearrowleft\rangle\right\rangle,
$$

corresponding to spin states $|1\rangle,|0\rangle,|-1\rangle$. Here $U$ and $\circlearrowright$ represent left and right circularly polarized photons. The biphoton is usually created via a nonlinear down conversion process in a neutrally polarized state like the second one in the above triplet.

For the biphoton system the concurrence $c(\psi)$ is closely related to its degree of polarization $P(\psi)=\sqrt{1-c(\psi)^{2}}$, that can be literally seen in classical polarization dependent 
intensity measurements [19]. In contrast, the quantity $|\langle\ell \mid \psi\rangle|^{2}=1-\left\langle\psi\left|S_{\ell}^{2}\right| \psi\right\rangle$ that enters into the pentagram inequality (7) requires a quantum measurement in a specific setting of the Hanbury Brown-Twiss interferometer described below.

The directions $\ell$ for the biphoton should be taken in the polarization space $\mathbb{R}_{\mathrm{pol}}^{3}$ with Stokes parameters $S_{1}, S_{2}, S_{3}$ as coordinates, rather than in the physical space $\mathbb{E}^{3}$. The Hilbert state space of a biphoton is obtained by complexification of the polarization space. The neutrally polarized states correspond to real state vectors $\ell \in \mathbb{R}_{\mathrm{pol}}^{3}$ that can be interpreted as follows. Let $P, Q$ be orthogonal polarization states of a photon described by the antipodal points $\pm \ell$ of the Poincaré sphere $\mathbb{S}^{2} \subset \mathbb{R}_{\mathrm{pol}}^{3}$. Then

$$
\ell=\frac{1}{\sqrt{2}}(|P Q\rangle+|Q P\rangle)
$$

In this setting the quantity $|\langle\ell \mid \psi\rangle|^{2}$ is equal to the coincidence rate in the Hanbury Brown-Twiss interferometer feeded by biphotons in state $\psi$ while polarization filters inserted into its arms select photons in orthogonal polarization states given by the antipodal points $\pm \ell$ of the Poincaré sphere.

As we have seen above, to test classical realism for a neutrally polarized state $\psi$ by a regular pentagram one needs the coincidence rate for a single direction $\ell$ such that $|\langle\ell \mid \psi\rangle|^{2}=\cos ^{2} \widehat{\ell \psi}=1 / \sqrt{5}$, which corresponds to the angle $\delta=\widehat{\ell \psi} \approx 0.8383$ radian. Quantum theory predicts the coincidence rate $1 / \sqrt{5} \approx 0.4472$, while to refute hidden variables one needs the rate greater than 0.4. However, the available raw experimental data from Fig. 8 of Ref. [20], by some reason fall far below the theoretical curve in a vicinity of $\delta=0.8383$ and provide no evidence for violation of classical realism in the biphoton system. The data clearly lack the required precision.

Recently, nonclassical behavior has been detected in a local two-qubit system formed by a single particle spin and two of its spatial modes created by a beam splitter [21]. Since nonlocality here is not an issue, the authors interpret the result as a test of noncontexuality. This may be an underestimation: a violation of $\mathrm{CHSH}$ inequality refutes any hidden parameters, context free or not [6].

In conclusion, we close the gap between twodimensional quantum systems, admitting hidden variables description [2], and four-dimensional systems that are incompatible with such a model [6] by constructing a Bell's type inequality for a three-dimensional spin- 1 system. We shortly discuss some physical implications and an underlying cause of the nonclassical behavior in spin-1 systems, as well as a perspective of its experimental verification.

This work was partially supported by Institute of Materials Science and Nanotechnology (UNAM),
Institute of Theoretical and Applied Physics (ITAP), and TÜBİTAK.

\footnotetext{
*klyachko@fen.bilkent.edu.tr

†can@fen.bilkent.edu.tr
}

[1] A. K. Ekert, Phys. Rev. Lett. 67, 661 (1991); C. Bennett, G. Brassard, C. Crepeau, R. Jozsa, A. Peres, and W. K. Wootters, Phys. Rev. Lett. 70, 1895 (1993); C. H. Bennett, G. Brassard, S. Popescu, B. Schumacher, J. A. Smolin, and W. K. Wootters, Phys. Rev. Lett. 76, 722 (1996); C. H. Bennett and S. J. Wiesner, Phys. Rev. Lett. 69, 2881 (1992); R. Jozsa and N. Linden, Proc. R. Soc. A 459, 2011 (2003).

[2] J. S. Bell, Physics (Long Island City, N.Y.) 1, 195 (1964); J. S. Bell, Rev. Mod. Phys. 38, 447 (1966).

[3] A. Aspect, J. Dalibard, and G. Roger, Phys. Rev. Lett. 49, 1804 (1982).

[4] M. Genovese, Phys. Rep. 413, 319 (2005).

[5] S. Kochen and E.P. Specker, J. Math. Mech. 17, 59 (1967); N. D. Mermin, Rev. Mod. Phys. 65, 803 (1993).

[6] A. Fine, Phys. Rev. Lett. 48, 291 (1982).

[7] Distributions with Given Marginals and Statistical Modelling, edited by C.M. Cuadras, J. Fontiana, and J. A. Rodriguez-Lallena (Kluwer Academic, Dordrecht, 2002).

[8] N. N. Vorob'ev, Theory Probab. Appl. 7, 147 (1962); H. G. Kellerer, Z. Wahrscheinlichkeitstheor. verw. Geb. 3, 247 (1964).

[9] A. Klyachko, arXiv:quant-ph/0206012.

[10] M. Franz, CONVEX, a Maple package for convex geometry, http://www-fourier.ujf-grenoble.fr/ franz/convex/.

[11] J. F. Clauser, M. A. Horne, A. Shimony, and R. A. Holt, Phys. Rev. Lett. 23, 880 (1969).

[12] E. Majorana, Nuovo Cimento 9, 43 (1932).

[13] What's in a name? That which we call a rose by any other name would smell as sweet. (Shakespeare, Romeo and Juliet, II, ii, 1-2).

[14] H. Barnum et al., Phys. Rev. A 68, 032308 (2003); L. Voila and H. Barnum, arXiv:quant-ph/0701124.

[15] A. A. Klyachko, in Proceedings of the NATO-ASI Conference on Physics and Theoretical Computer Science, edited by J.-P. Gazeau et al. (IOS Press, Amsterdam, 2007);

[16] W. K. Wootters, Phys. Rev. Lett. 80, 2245 (1998).

[17] M. A. Can, A. A. Klyachko, and A. S. Shumovsky, J. Opt. B 7, L1 (2005).

[18] Y. I. Bogdanov et al., Phys. Rev. Lett. 93, 230503 (2004); B. P. Lanyon et al., Phys. Rev. Lett. 100, 060504 (2008).

[19] M. V. Chekhova et al., Phys. Rev. A 70, 053801 (2004).

[20] L. A. Krivitskii et al., JETP 100, 521 (2005).

[21] M. Michler, H. Weinfurter, and M. Żukowski, Phys. Rev. Lett. 84, 5457 (2000); Y. Hazegawa et al., Nature (London) 425, 45 (2003); S. Basu et al., Phys. Lett. A 279, 281 (2001). 